\title{
Lack of Evidence for Sprouting of A $\beta$ Afferents into the Superficial Laminas of the Spinal Cord Dorsal Horn after Nerve Section
}

\author{
David I. Hughes, Dugald T. Scott, Andrew J. Todd, and John S. Riddell \\ Spinal Cord Group, Institute of Biomedical and Life Sciences, University of Glasgow, Glasgow G12 8QQ, United Kingdom
}

The central arborizations of large myelinated cutaneous afferents normally extend as far dorsally as the ventral part of lamina II in rat spinal cord. Woolf et al. (1992) reported that after nerve injury some of these afferents sprouted into lamina I and the dorsal part of lamina II, and it has been suggested that this could contribute to allodynia associated with neuropathic pain. Part of the evidence for sprouting was on the basis of the use of cholera toxin B subunit as a selective tracer for A-fibers, and the validity of this approach has recently been questioned; however, sprouting was also reported in experiments involving intra-axonal labeling of chronically axotomized afferents.

We have used intra-axonal labeling in the rat to examine central terminals of 58 intact sciatic afferents of presumed cutaneous origin and 38 such afferents axotomized 7-10 weeks previously. Both normal and axotomized populations included axons with hair follicle afferent-like morphology and arbors that entered the ventral half of lamina II; however, none of these extended farther dorsally. We also performed bulk labeling of myelinated afferents by injecting biotinylated dextran into the lumbar dorsal columns bilaterally $8-11$ weeks after unilateral sciatic nerve section. We observed that both ipsilateral and contralateral to the sectioned nerve, arbors of axons with hair follicle afferent-like morphology in the sciatic territory extended only as far as the ventral half of lamina II. Therefore these results do not support the hypothesis that $\mathrm{A} \beta$ afferents sprout into the superficial laminas after nerve section.

Key words: neuropathic pain; allodynia; nerve injury; myelinated afferent; low-threshold mechanoreceptor; intra-axonal injection

\section{Introduction}

Injury to peripheral nerves frequently leads to neuropathic pain. This can involve tactile allodynia (the perception of innocuous stimuli as painful), which is mediated by large myelinated $(\mathrm{A} \beta)$ afferents (Campbell et al., 1988; Koltzenburg et al., 1994). Because stimulation of these afferents does not normally produce pain, it is believed that changes within the CNS must be involved (Woolf, 1997).

Low-threshold $\mathrm{A} \beta$ mechanoreceptive afferents arborize in laminas III-V of the dorsal horn in the cat (Rexed, 1952; Brown, 1981), whereas in the rat, their arbors can extend into inner lamina II (IIi) (Woolf, 1987). Woolf and colleagues (Woolf et al., 1992; Shortland and Woolf, 1993) reported that after nerve injury, $A \beta$ afferents in the rat sprouted dorsally into the outer part of lamina II (IIo) and lamina I, areas that normally receive nociceptive input. They proposed that this could result in sensory miscoding, with cells that normally responded to noxious stimuli being activated by tactile input. The evidence for sprouting was on the basis of two experimental approaches: transganglionic transport of cholera toxin B subunit (CTb) and intra-axonal labeling. CTb injected into peripheral somatic nerves is normally

Received June 30, 2003; revised Aug. 29, 2003; accepted Aug. 29, 2003.

This work was supported by the Wellcome Trust. We thank R. Kerr, B. Finney, C. Watt, and M. McGill for expert technical assistance and Prof. D. J. Maxwell for helpful discussion and advice.

Correspondence should be addressed to Dr. J. S. Riddell, Spinal Cord Group, West Medical Building, University of Glasgow, University Avenue, Glasgow G12 8QQ, UK. E-mail: j.riddell@bio.gla.ac.uk.

Copyright $\odot 2003$ Society for Neuroscience $\quad$ 2270-6474/03/239491-09\$15.00/0 taken up selectively by myelinated afferents and transported to laminas I and IIi-V of the dorsal horn, with minimal labeling in lamina IIo (Robertson and Grant, 1985; LaMotte et al., 1991; Woodbury et al., 2000). Woolf et al. (1992) reported that if CTb was applied to sural nerves that had been injured 2 weeks previously, labeling was found in a continuous band from laminas I to $\mathrm{V}$, and they concluded that this was attributable at least partly to dorsal sprouting of $A \beta$ afferents. Two recent studies, however, have cast doubt on this explanation. Tong et al. (1999) found that many more dorsal root ganglion cells were labeled when CTb was applied to a chronically sectioned nerve and suggested that $\mathrm{CTb}$ may be transported by damaged unmyelinated afferents. Bao et al. (2002) reported that if CTb was injected into a sciatic nerve that was subsequently transected, labeling in the dorsal horn 2 weeks later was similar to that seen after application of CTb to an intact nerve. This indicates that the altered pattern of CTb labeling in the dorsal horn after nerve injury does not provide reliable evidence of sprouting and suggests that little if any dorsal sprouting occurs within the first 2 weeks after nerve injury.

It remains possible, however, that sprouting of $A \beta$ afferents occurs over a longer period, because Woolf and colleagues (Woolf et al., 1992; Shortland and Woolf, 1993) performed their intra-axonal labeling experiments on afferents transected 6-9 weeks previously. Koerber et al. $(1994,1999)$ also reported that chronically transected myelinated primary afferents in the cat arborized extensively in laminas I-IIo and interpreted this as evidence of sprouting. Because of recent doubts over whether 
sprouting of $\mathrm{A} \beta$ afferents occurs, we have reassessed the issue by performing intra-axonal labeling in rats with chronically sectioned sciatic nerves. We also looked for evidence of sprouting by injecting tracer into the dorsal columns to label collaterals of myelinated primary afferents.

\section{Materials and Methods}

All experiments were approved by the Ethical Review Process Applications Panel of the University of Glasgow and performed in accordance with the UK Animals (Scientific Procedures) Act 1986.

Intra-axonal labeling. Eight adult male Sprague Dawley rats (Harlan, Loughborough, UK; 240-290 gm) were anesthetized with halothane. Using sterile precautions, the left sciatic nerve was exposed at mid-thigh level, and a $5 \mathrm{~mm}$ length of the nerve proximal to its trifurcation was removed between two ligatures. All animals made an uneventful recovery, and between 7 and 10 weeks later they were prepared for in vivo electrophysiological recording and intra-axonal labeling. Electrophysiological recording experiments were also performed on eight agematched, unoperated rats to provide a sample of intra-axonally injected intact afferent fibers.

Anesthesia was induced with halothane and subsequently maintained by regular doses of sodium pentobarbitone (Rhône-Mérieux, Harlow, Essex, UK; $10 \mathrm{mg} / \mathrm{kg}$, i.v.), given as required. The depth of anesthesia was assessed by monitoring the pedal withdrawal reflexes, the corneal reflex, and blood pressure. During recording, the animals were paralyzed with Pancuronium bromide (Pavulon, Faulding, Leamington Spa, UK; 0.3 $\mathrm{mg} / \mathrm{kg}$, i.v., every $40 \mathrm{~min}$ ) and artificially ventilated. Anesthetic was then administered at a frequency commensurate with that required before paralysis, and the adequacy of anesthesia was checked by monitoring blood pressure and the absence of changes in response to noxious stimuli. Core temperature was maintained close to $38^{\circ} \mathrm{C}$ and that in paraffin pools covering the spinal cord and peripheral nerves at $35-37^{\circ} \mathrm{C}$. Mean blood pressure was always $>80 \mathrm{mmHg}$ and $\mathrm{P}_{\mathrm{CO}_{2}}$ was maintained within the range $4.0-4.5 \%$. Both sciatic nerves were placed on bipolar electrodes for stimulation, and a laminectomy was performed to expose the spinal cord from L3 to L6 segments. A silver ball electrode was placed on the dorsal columns to record afferent volleys and cord dorsum potentials (CDPs) evoked by sciatic nerve stimulation. Sharp, glass microelectrodes (1.0-1.5 $\mu \mathrm{m}$ tip diameter, $10-20 \mathrm{M} \Omega$ ) filled with a $2 \%$ solution of Neurobiotin (Vector Laboratories, Peterborough, UK) in $0.05 \mathrm{M}$ Tris- $\mathrm{HCl}$ buffer, $\mathrm{pH} 7.4$, containing $0.5 \mathrm{M} \mathrm{KCl}$ were inserted into the dorsal columns to impale single afferent fibers that were driven orthodromically from the sciatic nerve and conducted in the $\mathrm{A} \beta$ range (see below). Stimuli (0.2 msec duration) supramaximal for evoking A-fiber volleys and CDPs were used to search for afferent fibers. For intact nerves the stimulus intensity was $200 \mu \mathrm{A}$, whereas for sectioned nerves stimuli of $500 \mu \mathrm{A}$ were used because of the reduced excitability of axotomized fibers. In nerve-sectioned rats, all injected afferents were from the left (sectioned) side, whereas in unoperated rats afferents in either left or right sciatic nerve were injected. To increase the yield of labeled afferents, attempts were made to label several afferents in each animal. We did not investigate the peripheral receptive fields in the experiments involving intact afferents, because it would not be possible to relate receptive field properties to a particular labeled axon. After successful impalement, Neurobiotin was injected by ionophoresis $(10-15 \mathrm{nA}$ continuous positive current; 60-120 nA.min in total). Animals were maintained under general anesthesia for at least $1 \mathrm{hr}$ after the last attempted injection and then were perfused through the left ventricle with $4 \%$ freshly depolymerized formaldehyde. The conduction distance between stimulating electrodes on the sciatic nerve and the recording patch were measured and used together with conduction latencies for orthodromic impulses recorded during the experiment to calculate the conduction velocities of afferent fibers.

A $9 \mathrm{~mm}$ length of spinal cord centered on the injection site was removed and divided into three blocks that were postfixed overnight. The right side of each block was notched to allow subsequent orientation, and these were then cut into $60-\mu \mathrm{m}$-thick transverse sections with a vibratome. Sections were immersed in $50 \%$ ethanol for 30 min to enhance antibody penetration (Llewellyn-Smith and Minson, 1992) and incubated for $3 \mathrm{~d}$ at $4^{\circ} \mathrm{C}$ in rabbit anti-PKC $\gamma$ (Santa Cruz Biotechnology, Santa Cruz, CA; 1:1000) and streptavidin conjugated to lissamine rhodamine (Jackson ImmunoResearch, West Grove, PA; 1:1000), followed by donkey anti-rabbit IgG conjugated to Cy5 (Jackson ImmunoResearch; 1:100). Antibodies were made up in PBS containing 0.3\% Triton X-100. Sections were mounted in Vectashield (Vector Laboratories) and stored at $-20^{\circ} \mathrm{C}$.

All sections were initially viewed with a Nikon Eclipse 600 epifluorescence microscope and rhodamine filter set to identify axonal arborizations that approached the lamina II-III border. All of the sections containing arborizations of this type were then scanned with a Bio-Rad MRC1024 confocal laser scanning microscope equipped with a kryptonargon laser (Bio-Rad, Hemel Hempstead, UK). Sequential scanning of these sections was performed with the 568 and $647 \mathrm{~nm}$ lines (to reveal rhodamine and Cy5, respectively) through dry $(10 \times, 20 \times)$ and oilimmersion $(40 \times, 60 \times)$ objective lenses. The sections were also scanned using transmitted light through a dark-field condenser with the $10 \times$ lens. Projections of confocal image stacks were used to determine the proportion of afferents that had collaterals that passed from lamina III across the lamina II-III border. This was defined as the ventral border of the dense band of PKC $\gamma$ immunoreactivity (Polgár et al., 1999) and by the change in density of myelinated axons that can be seen with dark-field optics. For each afferent that had collaterals with branches that crossed the lamina II-III border, the total number of labeled varicosities in lamina II was counted. We also determined the proportion of these varicosities within lamina II that contacted PKC $\gamma$-immunoreactive cell bodies or dendrites by analyzing confocal images obtained through the $60 \times$ lens with Neurolucida for Confocal software (MicroBrightField, Inc., Colchester, VT).

Dorsal column injections. Four adult male Sprague Dawley rats (280$320 \mathrm{gm}$ ) were anesthetized with halothane and had the left sciatic nerve transected as described above. Between 8 and 11 weeks later, they were anesthetized with halothane and given bilateral injections of $20 \%$ biotinylated dextran (BD; 10,000 molecular weight, lysine-fixable; Molecular Probes, Eugene, OR) dissolved in distilled water directly into the dorsal columns at mid-lumbar level. A small slit was made in the dura as close as possible to the dorsal vein, and the micropipette was introduced with the tip angled at $10-15^{\circ}$ medially to a depth of at least $800 \mu \mathrm{m}$ below the pial surface. Between 100 and $200 \mathrm{nl}$ of $20 \%$ BD was pressure injected over $10-15 \mathrm{~min}$, during which time the pipette was progressively withdrawn to the surface. After a 3-5 d survival period, the rats were deeply anesthetized with pentobarbitone and perfused through the left ventricle with fixative containing $4 \%$ freshly depolymerized formaldehyde. Blocks of lumbar spinal cord on either side of the injection site were removed, postfixed overnight, notched to allow the two sides to be distinguished, and cut into $60 \mu \mathrm{m}$ transverse sections with a vibratome. Sections were incubated in streptavidin-rhodamine and reacted with antibodies to reveal PKC $\gamma$ as described above.

From the sample of four rats, three of the injections on the side ipsilateral to the nerve section and three of those on the contralateral side were judged to be successful; there was extensive labeling of axons within the dorsal columns and numerous well labeled axon collaterals with the typical appearance of myelinated cutaneous afferents were visible in the dorsal horn. The sciatic territory of the corresponding dorsal horn (LaMotte et al., 1991) was examined with the confocal microscope in seven vibratome sections from each of these experiments to determine whether BD-labeled arbors in lamina III with hair follicle afferent-like morphology entered the ventral or dorsal halves of lamina II. The sections were chosen on the basis of the presence of labeled axons that resembled the "flame-shaped arbors" (Scheibel and Scheibel, 1968), which are typical of $\mathrm{A} \beta$ hair follicle afferents (Brown, 1981; Woolf, 1987). They were scanned to reveal rhodamine-labeled afferents and $\mathrm{PKC} \gamma$ immunoreactivity through a $60 \times$ oil-immersion objective lens, and overlapping z-series ( 1 $\mu \mathrm{m} z$-separation) through the full thickness of each section were produced. For each vibratome section, the resulting z-series covered the mediolateral extent of the sciatic territory of the dorsal horn and included the dorsal part of lamina III and lamina II.

The confocal images were analyzed by using Neurolucida for Confocal software. Initially, the dorsal and ventral limits of the PKC $\gamma$ plexus were 


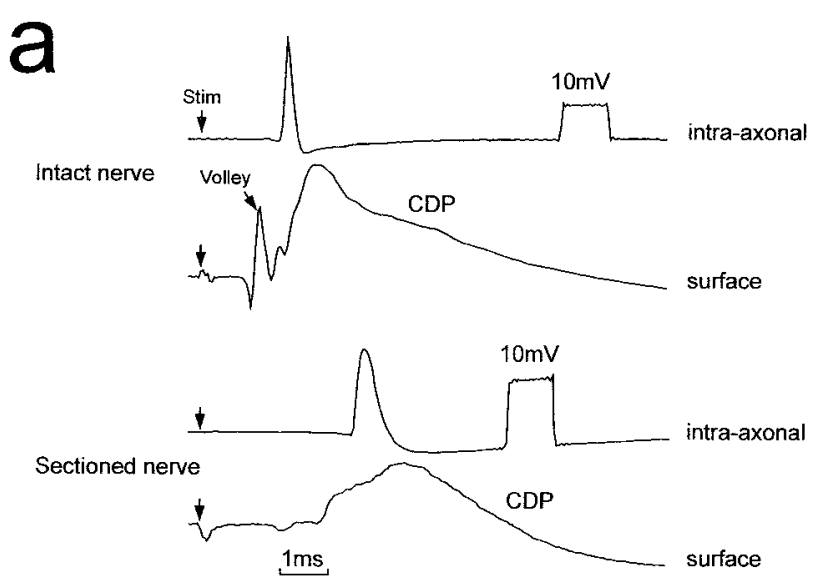

b

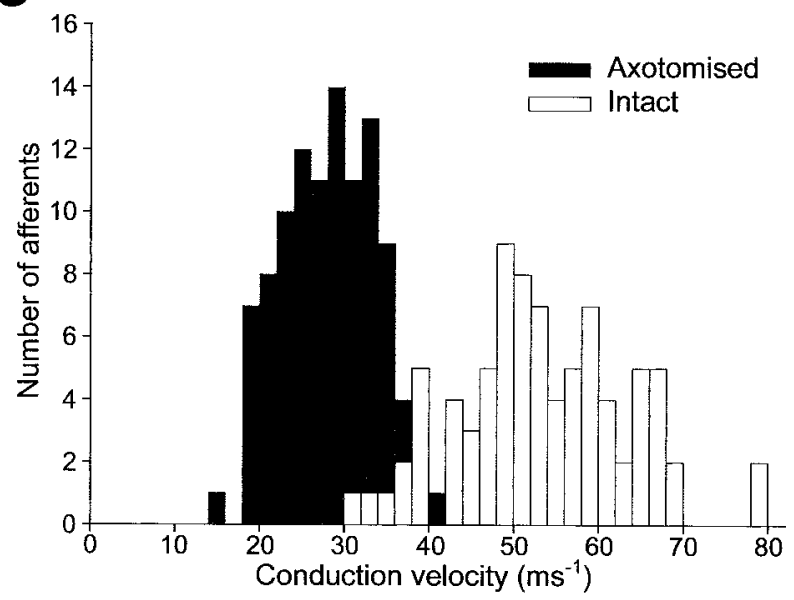

Figure 1. Intra-axonal recordings and conduction velocities of afferent fibers. $a$, Examples of recordings made from a rat with intact sciatic nerves (top) and from an animal in which the ipsilateral sciatic nerve was sectioned 7 weeks previously (bottom). The top trace of each pair is an intra-axonal recording. The bottom recordings are from the surface of the spinal cord (afferent volley and (DP). $b$, Histograms of conduction velocities for sciatic afferents of normal nerves $(n=82)$ and sectioned nerves $(n=108)$.

plotted onto drawings of the dorsal horn for each section. All of the BD-labeled arbors in lamina III that projected dorsally into the PKC $\gamma$ plexus were then identified, and all varicosities in lamina II arising from these arbors were counted.

\section{Results}

Conduction velocities of intra-axonally recorded afferents

Intra-axonal recordings were obtained from 82 afferents in rats with intact sciatic nerves and from 108 afferents in rats with chronically sectioned nerves. All of these afferents were identified by an orthodromic response to electrical stimulation of the sciatic nerve, and examples of recordings from a normal and an axotomized afferent fiber are shown in Figure 1a. Conduction velocities of the 82 afferents recorded from intact nerves ranged from 32 to $79 \mathrm{msec}^{-1}$ (Fig. $1 b$ ). The fibers with the faster conduction velocities are likely to have been Ia muscle afferents (Andrew et al., 1973; Riddell and Hadian, 1998), and some of the axons retrieved histologically had a morphology typical of these (see below). The conduction velocities of the 108 fibers recorded in animals with sectioned nerves were lower, ranging from 16 to 45 $\mathrm{msec}^{-1}$ (Fig. 1b), as would be expected for axotomized fibers (Cragg and Thomas, 1961).

\section{Morphology of intra-axonally labeled collaterals and} identification of laminar boundaries

Although we attempted to inject Neurobiotin into several afferents in each experiment, only some of these injections resulted in satisfactory labeling of the impaled axons and their collateral branches. Labeled axons were retrieved from all of the experiments, and in 15 of 16 experiments, more than one injected afferent was present. Labeled collaterals derived from a particular afferent could generally be distinguished from those belonging to other injected afferents in the same experiment, on the basis of their mediolateral position and laminar location within the dorsal horn (Brown, 1981). In this way, we were able to identify 58 well labeled sciatic afferents with laminar distributions that resembled those of cutaneous $A \beta$ afferents (Brown, 1981; Woolf, 1987) from rats with intact sciatic nerves and 38 such afferents from rats with chronically sectioned nerves. Six of the labeled axons from rats with intact nerves and nine of those from rats with sectioned nerves projected to motor nuclei and had a morphology typical of Ia afferents.

Labeled afferents that were identified as being of cutaneous origin had collateral arbors with a range of morphological appearances, presumably reflecting the different types of $\mathrm{A} \beta$ cutaneous afferent fiber within the sciatic nerve (Brown, 1981; Woolf, 1987). Within this group, we identified 27 afferents in intact preparations and 15 in rats with sectioned nerves that had recurrent axons and collaterals with flame-shaped arbors (Scheibel and Scheibel, 1968), typical of those belonging to identified A $\beta$ hair follicle afferents (Brown et al., 1977; Shortland et al., 1989) (Table 1). Only afferents with this flame-shaped morphology were found to have collaterals that approached the lamina II-III border, and therefore these were analyzed in detail to determine the dorsal extent of their arborizations. In several experiments, more than one labeled hair follicle-like afferent appeared to be present in the same dorsal horn (Fig. $4 a, b$ ). In these cases, labeled collaterals were invariably found in matching mediolateral positions in different sections in the series, and the position of these collaterals was used to assign them to a particular afferent (Brown, 1981).

The distribution of PKC $\gamma$ immunoreactivity was used to define the boundary between laminas II and III (Polgár et al., 1999). Although scattered immunoreactive cell bodies and dendrites were present in laminas III and IV, a plexus of PKC $\gamma$ immunoreactive dendrites and a high density of immunoreactive cell bodies occupied the ventral half of lamina II and extended as far ventrally as the lamina II-III boundary, as determined by dark-field microscopy (Fig. 2). Although the strength of PKC $\gamma$ immunostaining varied across the mediolateral extent of the dorsal horn (being stronger in the lateral part), the ventral limit of the plexus was found to provide a reliable indication of the lamina II-III border. Because we found that the band of PKC $\gamma$ immunoreactivity occupied the ventral half of lamina II (Fig. 2), we used its dorsal limit to determine whether collaterals entered the dorsal half of the lamina. The PKC $\gamma$ plexus was also clearly visible after nerve section and retained the same relation to lamina II, as defined by dark-field microscopy.

In the rats with intact sciatic nerves, most of the labeled axons with hair follicle afferent-like morphology (18 of 27) had collaterals that arborized extensively in lamina III but did not cross the lamina II-III border (Fig. $3 a-c$ ). Collaterals from the other nine afferents of this type entered lamina II (Fig. $3 d-f$ ), and these gave rise to between 14 and 812 (mean $299 \pm 282$ SD) varicosities per afferent within this lamina (Table 1). Although some of these collaterals extended as far as the dorsal limit of the PKC $\gamma$ plexus 
Table 1. Quantitative data from intra-axonal injection experiments

\begin{tabular}{|c|c|c|c|c|c|}
\hline & $\begin{array}{l}\text { Number of } \\
\text { cutaneous-type } \\
\text { afferents }\end{array}$ & $\begin{array}{l}\text { Number of HFA-like } \\
\text { axons (\% of } \\
\text { cutaneous-type) }\end{array}$ & $\begin{array}{l}\text { Number of HFA-like axons } \\
\text { entering ventral half of } \\
\text { lamina } \text { Il }^{a}\end{array}$ & $\begin{array}{l}\text { Mean number of boutons per } \\
\text { afferent in ventral lamina II } \\
\text { (range) }\end{array}$ & $\begin{array}{l}\text { Mean } \% \text { of boutons per afferent } \\
\text { that contacted PKC } \gamma \text { profiles in } \\
\text { lamina II (range) }\end{array}$ \\
\hline Normal afferents & 58 & 27 (46.6) & 9 & $299(14-812)$ & $4.7(0-7.9)$ \\
\hline Axotomized afferents & 38 & $15(39.5)$ & 5 & $254(8-670)$ & $5.4(0-12.2)$ \\
\hline
\end{tabular}

HFA, Hair follicle afferent.

${ }^{a}$ Only axons with HFA-like morphology had collaterals that arborized in ventral lamina II, and none of these entered the outer part of lamina II or lamina I
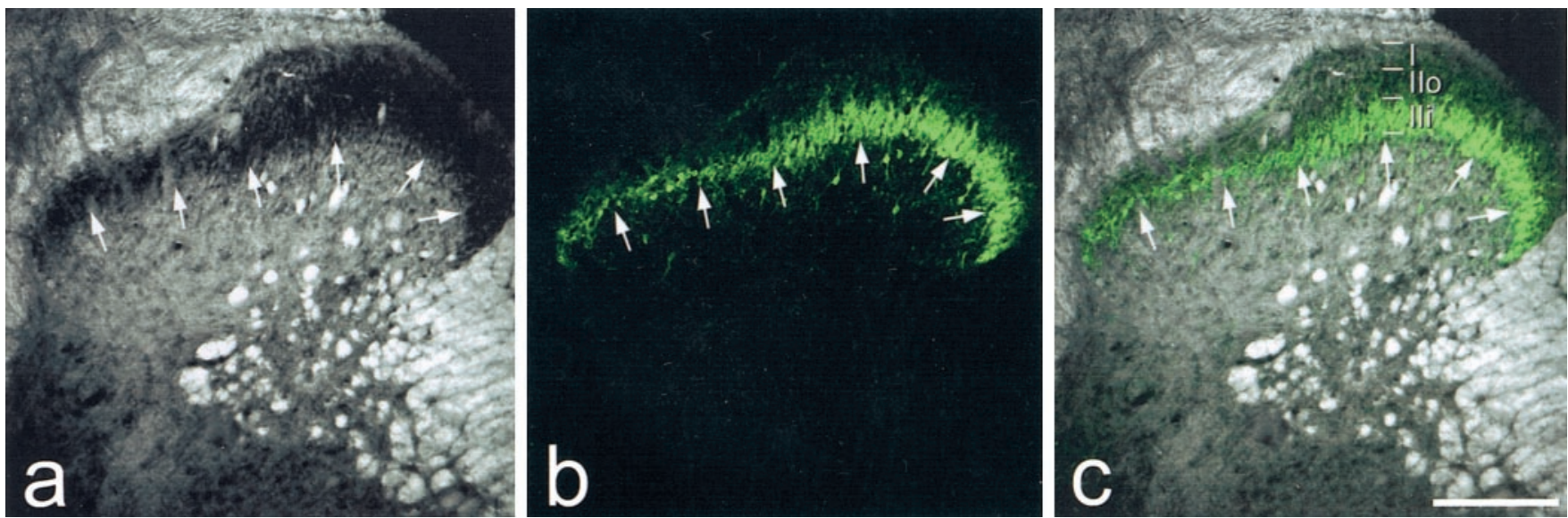

Figure 2. Confocal images showing the laminar location of PKC $\gamma$ immunostaining in the dorsal horn of a rat with intact sciatic nerves. In $a$ the dorsal horn has been scanned with transmitted light through a dark-field condenser, whereas PKC $\gamma$ immunoreactivity is seen in $b$ and the two images are merged in $c$. In the dark-field scan, lamina II appears dark because of the lack of myelin, and its ventral border (arrows) is clearly visible. The ventral edge of the band of PKC $\gamma$ immunostaining corresponds closely to the lamina II-III border defined with dark-field illumination. The dorsal limit of this band was used to distinguish inner and outer halves of lamina II (lli and Ilo). Scale bar, $200 \mu \mathrm{m}$.
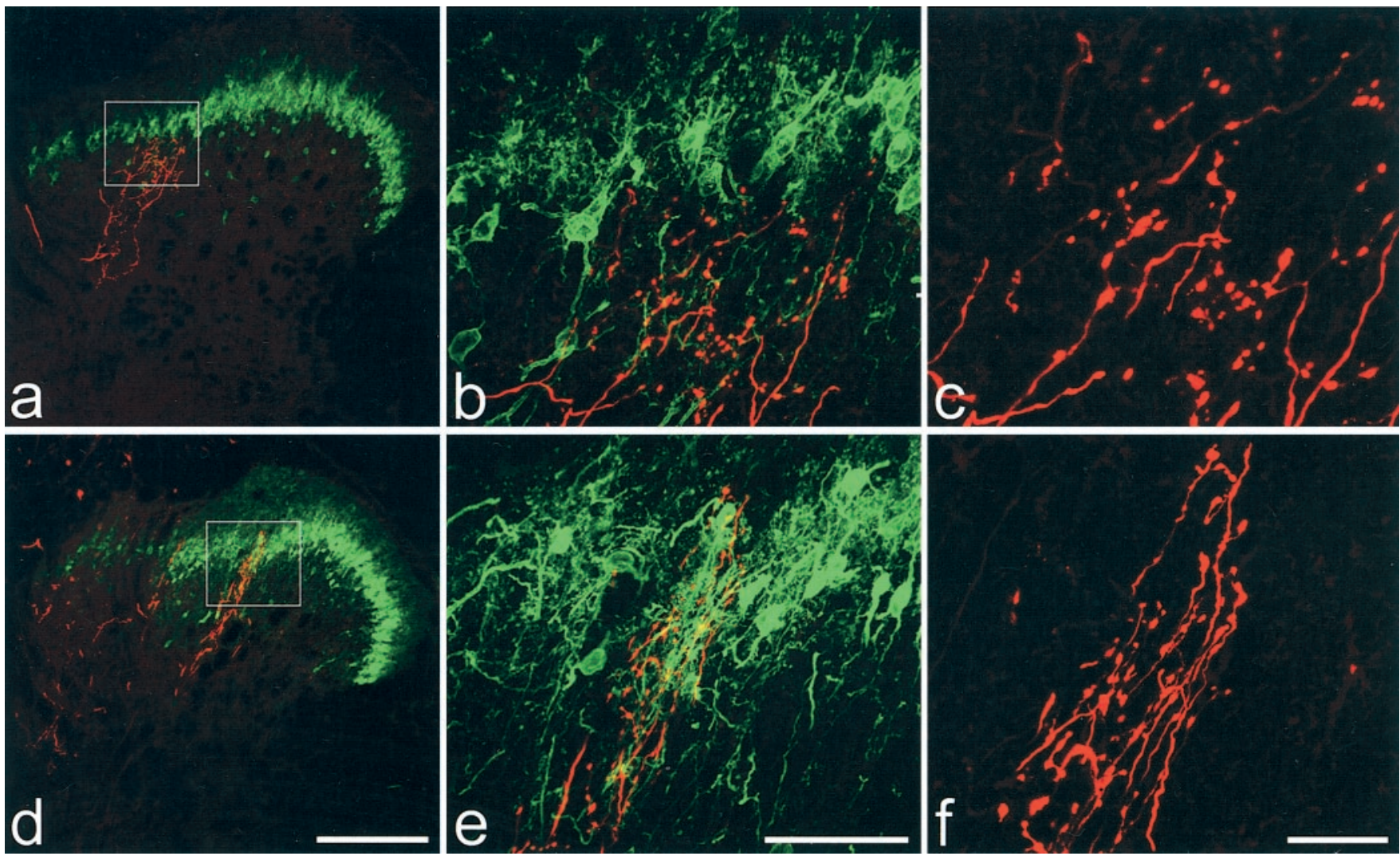

Figure 3. Two intra-axonally injected collaterals with flame-shaped arbors in rats with intact sciatic nerves. In each case, the injected afferent appears red, and PKC $\gamma$ immunoreactivity is green. Boxes in $a$ and $d$ correspond to the areas shown in $b$ and e. $a$-c show a collateral that extends as far dorsally as the lamina II-III border (defined as the ventral limit of the PKC $\gamma$-immunoreactive plexus) but does not enter it. $d$-fshow another collateral that extends through the full thickness of the PKC $\gamma$ plexus. Scale bars: $a, d, 200 \mu \mathrm{m} ; b, e, 50 \mu \mathrm{m} ; c, f, 20 \mu \mathrm{m}$. Images $a$ and $d$ were created from projections of 22 and 24 optical sections at $4 \mu \mathrm{m} z$-separation, respectively. $b, c, e$, and $f$ are projections of 42,59, 42, and 61 optical sections at $1 \mu \mathrm{m} z$-separation, respectively. 

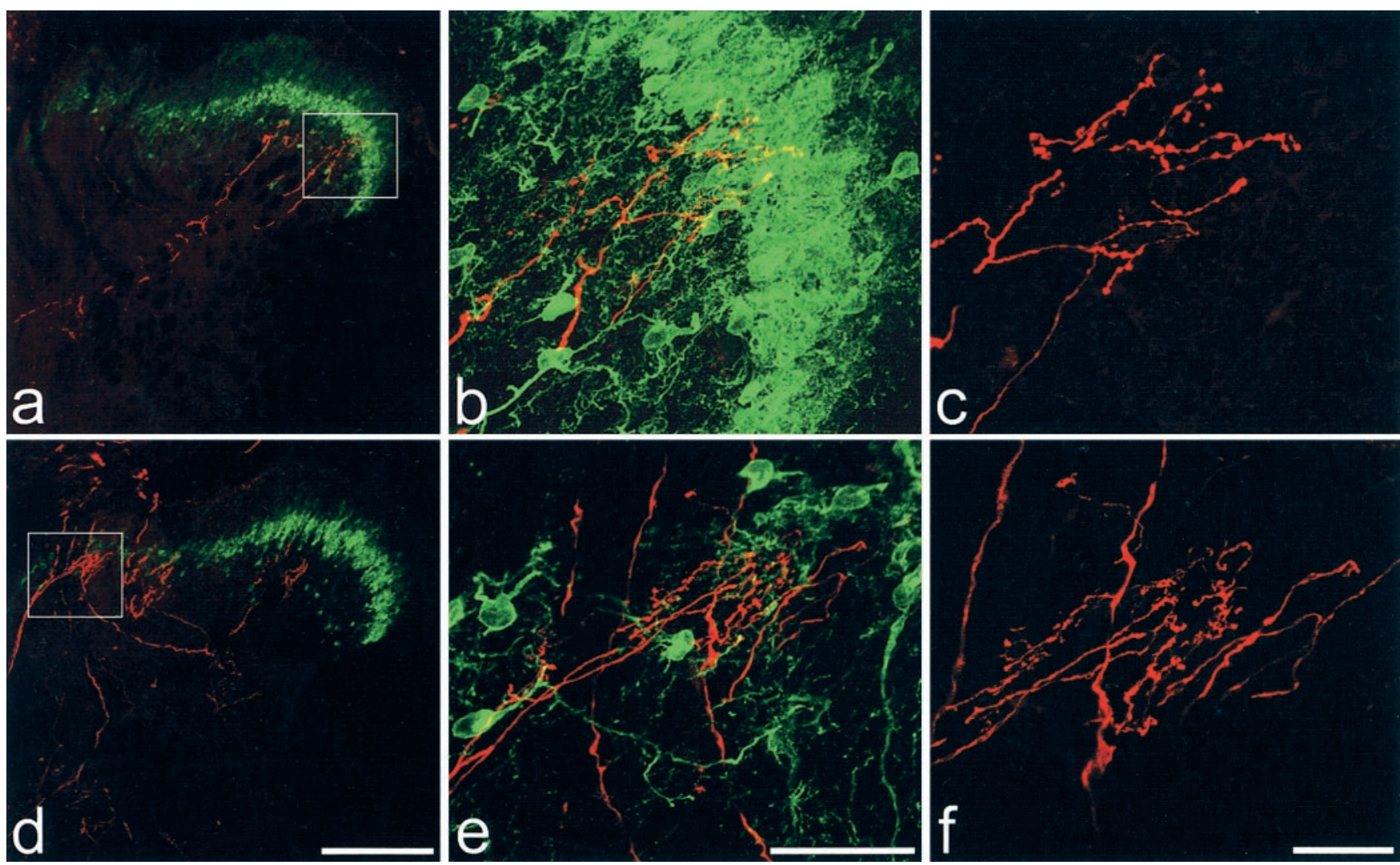

Figure 4. Two intra-axonally injected collaterals with flame-shaped arbors in rats with chronically sectioned sciatic nerves. In each case, the injected afferent is red, and PKC $\gamma$ immunoreactivity is green. The collateral shown in $a-c$ (from a rat that survived 7 weeks after nerve section) extends approximately halfway through the thickness of the PKC $\gamma$ plexus but does not enter the outer half of lamina II. The collateral in $d-f$ (from a rat that survived 8 weeks after nerve section) also extends into, but not through, the PKC $\gamma$ plexus. In this region the plexus is partly disrupted by one of the bundles of myelinated afferents that pass through the medial part of the superficial dorsal horn. Boxes in $a$ and $d$ correspond to the areas shown in $b$ and $e$. Note that in each section, parts of other labeled collaterals are also visible. Scale bars: $a, d, 200 \mu \mathrm{m} ; b, e, 50 \mu \mathrm{m} ; c, f, 20 \mu \mathrm{m}$. Images $a$ and $d$ were created from projections of 22 and 20 optical sections at $4 \mu \mathrm{m} z$-separation, respectively. $b, c, e$, and $f$ are projections of $55,51,55$, and 36 optical sections at $1 \mu \mathrm{m} z$-separation, respectively.

(Fig. 3d,e), none entered the dorsal half of lamina II (i.e., the area dorsal to the plexus). For most of these afferents, some of the labeled varicosities in lamina II formed contacts with PKC $\gamma$ immunoreactive dendrites or cell bodies: the proportion of varicosities that did so varied from 0 to $7.9 \%$ (mean $4.7 \pm 2.7 \% \mathrm{SD}$ ) (Table 1).

Similar results were obtained from rats with chronically sectioned sciatic nerves. Ten of the 15 afferents with hair follicle-like morphology had collaterals that arborized in lamina III but did not enter lamina II. The other five afferents of this type had collaterals that entered the ventral half of lamina II (Fig. 4), where they gave rise to between 8 and 670 varicosities (mean $254 \pm 289$ ) (Table 1). None of the labeled collaterals entered the dorsal half of lamina II or extended into lamina I. Again, some of the varicosities in lamina II contacted PKC $\gamma$-immunoreactive dendrites or cell bodies (range $0-12.2 \%$; mean $5.4 \pm 5.7 \%$ ) (Fig. 5, Table 1)

\section{Central labeling after BD injection into the dorsal columns}

The injections of BD were restricted to the dorsal columns in three experiments but extended through lamina $\mathrm{X}$ and into the underlying white matter in one case (Fig. 6). In all cases, on the side corresponding to a successful BD injection, there was extensive labeling of axons in the dorsal columns and dorsal roots, as well as collateral and terminal labeling in most parts of the gray matter (Fig. 7). In addition, cell bodies were often present in laminas III-V of the dorsal horn, and presumably these included postsynaptic dorsal column neurons (Giesler et al., 1984). Many labeled axon collaterals were seen passing from the dorsal columns through the medial part of the dorsal horn toward lamina IV (Fig. 7a). Plexuses of BD-labeled axons that resembled flameshaped arbors were observed in lamina III, and these could often be followed into the ventral half of lamina II (Fig. $7 b, d$ ). Numerous varicosities were present along the course of these arbors (Fig. $7 b, d$, insets). In addition, scattered BD-labeled varicosities that were not attached to these arbors were present throughout the dorsal horn, including lamina I and the outer part of lamina II. These are likely to have arisen (at least in part) from corticospinal fibers that travel through the ventral part of the dorsal columns in the rat (Brown, 1971) and terminate throughout the gray matter, including laminas I and II (Casale et al., 1988). The same pattern of labeling was seen both contralateral and ipsilateral to the chronically sectioned sciatic nerve.

Because of the large number of axons that were labeled with $\mathrm{BD}$, it was not possible to identify individual afferents or collaterals in these sections. We therefore examined all arbors that extended from lamina III into lamina II, counted the number of varicosities that arose in lamina II, and pooled the results from the seven sections that were analyzed for each dorsal horn. On the intact side, the total numbers of varicosities counted in the ventral half of lamina II (i.e., within the PKC $\gamma$ plexus) in the three experiments were 732,655 , and 332 , whereas the corresponding values for the side ipsilateral to the sectioned sciatic nerve were 588,576 , and 327 . The BD-labeled arbors that originated in lamina III were never seen to project dorsal to the PKC $\gamma$ plexus (Fig. 

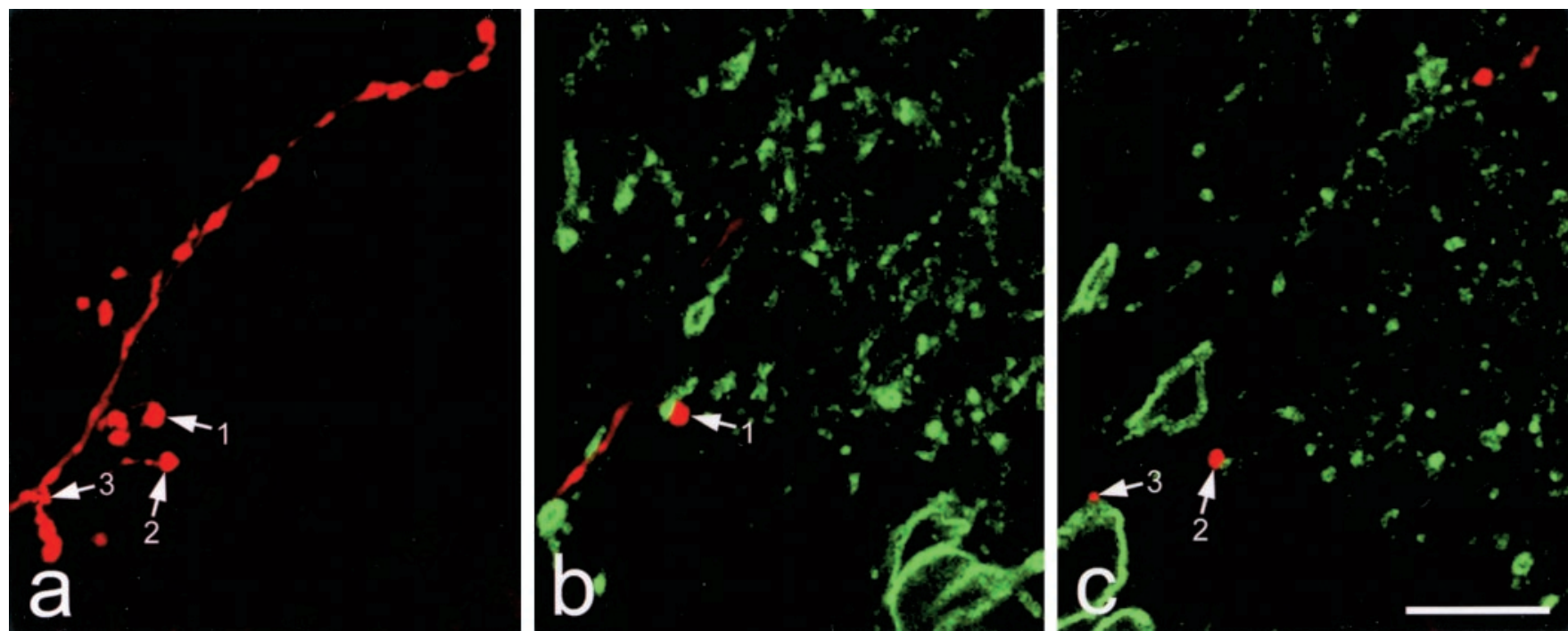

Figure 5. Contacts formed by an intra-axonally injected sciatic afferent (7 weeks after ipsilateral sciatic nerve section) and PKC $\gamma$-immunoreactive structures in the ventral half of lamina II. $a$ shows a projection of 50 confocal images at $1 \mu \mathrm{m} z$-separation scanned to reveal the labeled collateral. Several varicosities are present, and three of these are indicated with arrows. $b$ and $c$ show single optical sections from this series, which have been scanned to reveal the afferent (red) and PKC $\gamma$ immunoreactivity (green). Varicosities 1 and 2 contact small PKC $\gamma$-immunoreactive dendrites, and varicosity 3 is in contact with a PKC $\gamma$-immunoreactive cell body. Scale bar, $10 \mu \mathrm{m}$.

7), and no varicosities arising from these arbors were seen in the dorsal half of lamina II.

\section{Discussion}

In agreement with other studies in the rat (Woolf, 1987; Shortland et al., 1989; Shortland and Woolf, 1993), we found that although the major termination zone of afferents with hair follicle-like morphology was in lamina III, some of these axons had terminal arbors that extended into the ventral half of lamina II. Many neurons in ventral lamina II respond to brushing of the skin (Light and Willcockson, 1999), and some of these presumably receive monosynaptic input from $A \beta$ hair follicle afferents. In contrast to previous studies (Woolf et al., 1992; Shortland and Woolf, 1993), we found no evidence that chronically sectioned afferents of this type had arbors that extended more dorsally (i.e., into the dorsal half of lamina II or lamina I).

\section{Technical considerations}

To ensure that we labeled only axotomized afferents when recording intra-axonally in nerve-sectioned rats, fibers were identified by an orthodromic response to electrical stimulation of the sciatic nerve. The axons recorded from nervesectioned animals had lower conduction velocities than those in intact nerves (Fig. 1). Because a reduction in conduction velocity has been reported after peripheral axotomy (Cragg and Thomas, 1961), this provides further evidence that these fibers had been axotomized. In preliminary experiments, we found that it was considerably more difficult to impale afferents belonging to chronically sectioned nerves (possibly because of thinning of the parent axons in the dorsal columns) (Horch, 1976), and the proportion that were successfully injected with Neurobiotin and retrieved in histological sections was smaller than for intact affer-
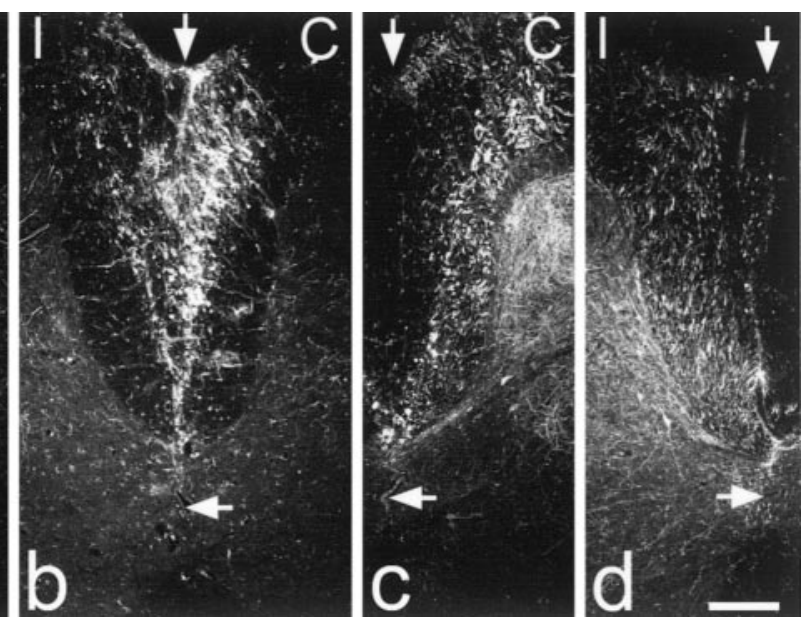

Figure 6. BD labeling in the dorsal columns. Sections of the spinal cord close to the injection sites are shown for the four experiments in which BD was injected into the dorsal columns. BD has been detected with streptavidin-rhodamine, and each image shows a single optical section scanned to reveal this with the confocal microscope. In each case the left side is ipsilateral (I) and the right side is contralateral $(C)$ to the left sciatic nerve, which had been transected between 8 and 11 weeks earlier. The top vertical arrow indicates the midline, and the bottom horizontal arrow indicates the position of the central canal. In $a$ and $b$ of the ventral white commissure. cand $d$ are from experiments in which only one injection was successful. In c, this was on the right (contralateral) side and in $d$ it was on the left (ipsilateral) side. Scale bar, $200 \mu \mathrm{m}$.

ents. To achieve a satisfactory yield of axotomized afferents, we therefore attempted to fill several axons in a single experiment and obtain matched samples of intact and axotomized fibers; we also labeled several fibers in each experiment on rats with intact sciatic nerves. The proportion of putative cutaneous afferents with flame-shaped arbors was similar in the normal and axotomized populations (27 of 58 and 15 of 38, respectively), which suggests that a comparable sample was labeled in each case. In contrast to previous studies in which horseradish peroxidase was used for intra-axonal labeling (Woolf et al., 1992; Shortland and Woolf, 1993; Koerber et al., 1994, 1999), we injected Neurobiotin, because this has been found to produce more extensive labeling of primary afferents (Wilson et al., 1996). The propor- 

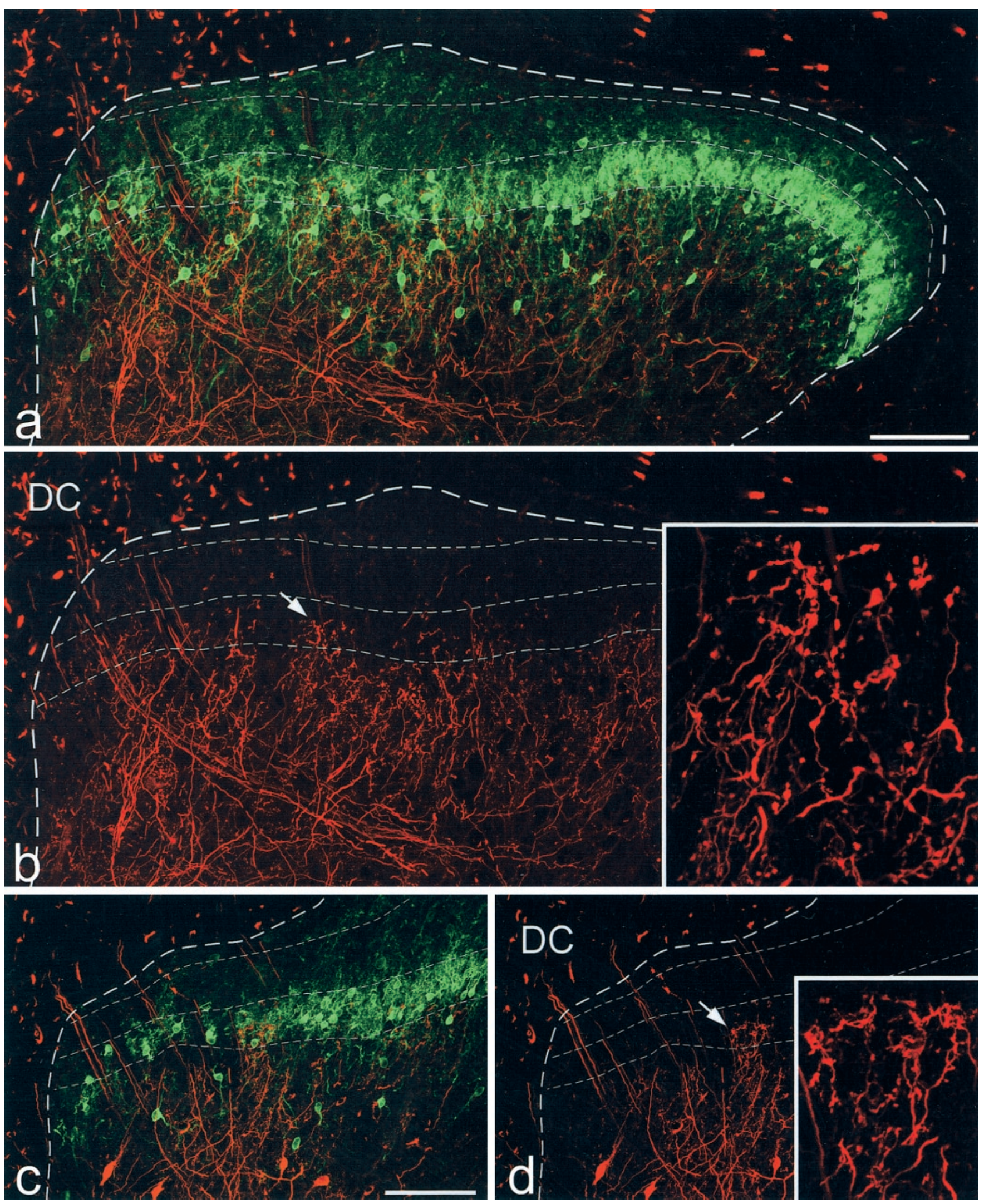

Figure 7. BD and PKC $\gamma$ immunoreactivity in sections from animals that had had BD injected into the dorsal columns. $a$ and $b$ show labeling resulting from the injection on the side with the sciatic nerve intact in the experiment illustrated in Figure $6 a$. Fine dashed lines show the extent of the PKC $\gamma$ plexus and also the approximate position of the lamina I-II border. In $b$ a terminal arborization (arrow) can be seen extending into the ventral half of lamina II. This is shown in detail in the inset. c and $d$ show the labeling resulting from injection of BD into the dorsal columns on the same side as a chronically sectioned sciatic nerve (cut 8 weeks before the injection) in the experiment illustrated in Figure $6 b$. Again, the boundaries of the PKC $\gamma$ plexus and the lamina I-II border are shown with fine dashed lines. A terminal arborization extends into the ventral half of lamina II (arrow) and is shown in detail in the inset. Scale bars, $100 \mu \mathrm{m}$. $a, b$ and $c, d$ are projections of 18 and 24 optical sections at $2 \mu \mathrm{m} z$-separation, respectively. Insets in $b$ and $d$ are projections of 30 and 34 optical sections at $1 \mu \mathrm{m} z$-separation, respectively. 
tion of axons with flame-shaped arbors that extended into lamina II, and also the numbers of varicosities in lamina II, were similar in the intact and axotomized afferents (Table 1), suggesting that the extent of filling of the two populations was comparable.

The intra-axonal labeling technique is inevitably biased toward sampling afferents with larger diameters. To investigate a broader range of $A \beta$ afferents, we therefore performed additional experiments in which BD was injected directly into the dorsal columns, where these axons are located. Because the dorsal columns also contain other types of axon, these injections resulted in labeling of other neuronal populations, including cell bodies of presumed postsynaptic dorsal column cells and terminals that probably arose from corticospinal axons; however, we were able to identify profuse axonal arborizations with varicosities in lamina III and the ventral half of lamina II, within the sciatic nerve territory (Fig. $7 b, d$ ). It is likely that these included hair follicle afferents, because bundles of large axons could be seen passing from the dorsal columns in a curving course that is characteristic of $\mathrm{A} \beta$ cutaneous fibers (Fig. $7 b$ ). As in the intra-axonal injection experiments, these arborizations were never seen to extend through the PKC $\gamma$ plexus into the dorsal half of lamina II, even when injections were ipsilateral to the chronically sectioned sciatic nerve.

Because the main aim of the study was to determine the dorsal extent of axotomized primary afferents, it was necessary to have a reliable means of identifying the lamina II-III border. We used PKC $\gamma$ because [as reported by Polgár et al. (1999)] its ventral limit exactly matched the II-III border as defined by dark-field microscopy (Fig. 2). In addition, unlike many other neurochemical markers, PKC $\gamma$ immunostaining did not show obvious changes after nerve section (Figs. 3, 4, 6). Although Miletic et al. (2000) reported a slight increase in the intensity of PKC $\gamma$ immunostaining in lamina II after chronic constriction of the sciatic nerve, they also found no significant alteration in the distribution of immunoreactivity. A further advantage of this approach was that the dorsal limit of the PKC $\gamma$ plexus, which lay approximately midway between the dorsal and ventral borders of lamina II, corresponded to the most dorsal extent of arborizations of intact afferents with flame-shaped arbors (Fig. $3 e$ ).

\section{Comparison with previous reports}

In an intra-axonal injection study in the rat, Woolf and colleagues (Woolf et al., 1992; Shortland and Woolf, 1993) reported that 8 of 26 axotomized sural $\mathrm{A} \beta$ afferents with hair follicle-like morphology had terminal arbors that extended into the outer part of lamina II or lamina I, an arrangement that was never observed normally (Shortland et al., 1989). Because we failed to detect dorsal sprouting in our intra-axonally injected axotomized afferents, it is necessary to consider whether methodological differences could account for these discrepancies. Although we used a postoperative survival time similar to that of Shortland and Woolf (1993) (7-10 weeks, compared with 6-12 weeks in their study), we examined afferents in a transected sciatic nerve rather than using the sural nerve (a branch of the sciatic). The sciatic nerve was chosen to increase the yield of labeled axons in each experiment, but its afferent fiber spectrum differs from that in the sural: it has a relatively lower proportion of hair follicle afferents (because its territory includes glabrous skin) and also contains proprioceptors. Our sample, however, included axons with hair follicle afferent-like morphology, and it seems unlikely that hair follicle afferents in the sural nerve would behave differently from those in other branches of the sciatic nerve. Our sample of 15 axotomized afferents with hair follicle-like morphology should have been sufficient to demonstrate any sprouting into the superficial laminas, because Shortland and Woolf (1993) reported this type of sprouting in approximately one-third ( 8 of 26) of these afferents.

In an in vitro neonatal mouse preparation, Woodbury and Koerber (2003) recently described a population of nociceptors with dorsally recurving collateral arbors that extended throughout laminas I-V of the dorsal horn and reported that these afferents became myelinated and retained this morphology in adulthood. If axons of this type also occur in other species, they may account for some of the myelinated afferents with collateral branches extending into lamina I that were identified in previous studies (Woolf et al., 1992; Shortland and Woolf, 1993; Koerber et al., 1994, 1999). We did not observe any axons with this morphology in our sample of injected afferents, and this may be because they are relatively infrequent or because they are more difficult to impale in an in vivo rat preparation than lowthreshold $\mathrm{A} \beta$ cutaneous afferents. Woodbury and Koerber (2003) noted that transganglionic labeling studies with CTb have failed to demonstrate myelinated primary afferents with arbors that extend throughout laminas I-V and suggested that this may be because of the diffuse nature of their arborizations. In our BD injection experiments, we analyzed only axons that resembled typical $\mathrm{A} \beta$ hair follicle afferents (i.e., those with a dense arborization in lamina III), because it has been suggested that these axons sprout dorsally after nerve injury (Woolf et al., 1992; Shortland and Woolf, 1993). If myelinated nociceptors with arbors that extend from laminas I to $\mathrm{V}$ exist in the adult rat, the diffuse nature of these arbors (Woodbury and Koerber, 2003) could account for our failure to recognize them in the $\mathrm{BD}$ injection experiments.

Although we cannot rule out the possibility that some hair follicle afferents do sprout dorsally into the outer half of lamina II, our failure to see axonal plexuses extending dorsal to the band of PKC $\gamma$ immunoreactivity in either the intra-axonal injection experiments or those in which BD was injected into the dorsal columns suggests that if such sprouting does occur, it is a rare event.

There is evidence from both c-fos expression studies and in vitro electrophysiology that transmission of tactile information conveyed by $\mathrm{A} \beta$ afferents to neurons in the superficial dorsal horn is enhanced after nerve injury (Molander et al., 1994; Bester et al., 2000; Kohama et al., 2000; Okamoto et al., 2001; Kohno et al., 2003); however, there are other routes through which $\mathrm{A} \beta$ input can reach the superficial laminas. Some $A \beta$ hair follicle afferents have arbors that enter lamina II (Shortland et al., 1989), and our evidence suggests that their postsynaptic targets may include PKC $\gamma$-immunoreactive cells, which are likely to be excitatory interneurons (Polgàr et al., 1999). In addition, certain lamina II neurons (e.g., stalked cells) have dendrites that enter lamina III, and because some of these send axons to lamina I, they may convey tactile information from $\mathrm{A} \beta$ afferents to projection neurons in this lamina (Gobel, 1978). The enhancement of tactile inputs to neurons in the superficial laminas after nerve injury could result from increased efficacy of glutamatergic transmission (Harris et al., 1996) or a reduction of GABAergic inhibition (Moore et al., 2002) in these pathways.

\section{References}

Andrew BL, Leslie GC, Thompson J (1973) Distribution and properties of muscle spindles in the caudal segmental muscles of the rat together with some comparisons with hindlimb muscle spindles. Q J Exp Physiol Cogn Med Sci 58:19-37.

Bao L, Wang HF, Cai H-J, Tong Y-G, Jin S-X, Lu Y-J, Grant G, Hökfelt T, Zhang X (2002) Peripheral axotomy induces only very limited sprouting 
of coarse myelinated afferents into inner lamina II of rat spinal cord. Eur J Neurosci 16:175-185.

Bester H, Beggs S, Woolf CJ (2000) Changes in tactile stimuli-induced behavior and c-Fos expression in the superficial dorsal horn and in parabrachial nuclei after sciatic nerve crush. J Comp Neurol 428:45-61.

Brown AG (1981) Organization in the spinal cord: the anatomy and physiology of identified neurones. Berlin: Springer.

Brown AG, Rose PK, Snow PJ (1977) The morphology of hair follicle afferent fibre collaterals in the spinal cord of the cat. J Physiol (Lond) 272:779-797.

Brown LT (1971) Projections and termination of the corticospinal tract in rodents. Exp Brain Res 13:432-450.

Campbell JN, Raja SN, Meyer RA, Mackinnon SE (1988) Myelinated afferents signal the hyperalgesia associated with nerve injury. Pain 32:89-94.

Casale EJ, Light AR, Rustioni A (1988) Direct projections of the corticospinal tract to the superficial laminae of the spinal cord in the rat. J Comp Neurol 278:275-286.

Cragg BG, Thomas PK (1961) Changes in conduction velocity and fibre size proximal to peripheral nerve lesions. J Physiol (Lond) 157:315-327.

Giesler GJ, Nahin RL, Madsen AM (1984) Postsynaptic dorsal column pathway in the rat. 1. Anatomical studies. J Neurophysiol 51:260-275.

Gobel S (1978) Golgi studies of the neurons in layer II of the dorsal horn of the medulla (trigeminal nucleus caudalis). J Comp Neurol 180:395-414.

Harris JA, Corsi M, Quartaroli M, Arban R, Bentivoglio M (1996) Upregulation of spinal glutamate receptors in chronic pain. Neuroscience $74: 7-12$.

Horch KW (1976) Ascending collaterals of cutaneous neurons in the fasciculus gracilis of the cat during peripheral nerve regeneration. Brain Res 117:19-32.

Koerber HR, Mirnics K, Brown PB, Mendell LM (1994) Central sprouting and functional plasticity of regenerated primary afferents. J Neurosci 14:3655-3671.

Koerber HR, Mirnics K, Kavookjian AM, Light AR (1999) Ultrastructural analysis of ectopic synaptic boutons arising from peripherally regenerated primary afferents. J Neurophysiol 81:1636-1644.

Kohama I, Ishikawa K, Kocsis JD (2000) Synaptic reorganization in the substantia gelatinosa after peripheral nerve neuroma formation: aberrant innervation of lamina II neurons by $\mathrm{A} \beta$ afferents. J Neurosci 20:1538-1549.

Kohno T, Moore KA, Baba H, Woolf CJ (2003) Peripheral nerve injury alters excitatory synaptic transmission in lamina II of the rat dorsal horn. J Physiol (Lond) 548.1:131-138.

Koltzenburg M, Torebjork HE, Wahren LK (1994) Nociceptor modulated central sensitization causes mechanical hyperalgesia in acute chemogenic and chronic neuropathic pain. Brain 117:579-591.

LaMotte C, Kapadia SE, Shapiro CM (1991) Central projections of the sciatic, saphenous, median and ulnar nerves of the rat demonstrated by transganglionic transport of choleragenoid-HRP (B-HRP) and wheat germ agglutinin-HRP (WGA-HRP). J Comp Neurol 311:546-562.

Light AR, Willcockson HH (1999) Spinal laminae I-II neurons in rat recorded in vivo in whole cell, tight seal configuration: properties and opioid responses. J Neurophysiol 82:3316-3326.

Llewellyn-Smith IJ, Minson JB (1992) Complete penetration of antibodies into vibratome sections after glutaraldehyde fixation and ethanol treatment: light and electron microscopy for neuropeptides. J Histochem Cytochem 40:1741-1749.

Miletic V, Bowen KK, Miletic G (2000) Loose ligation of the rat sciatic nerve is accompanied by changes in the subcellular content of protein kinase $\mathrm{C}$ beta II and gamma in the spinal dorsal horn. Neurosci Lett 288:199-202.

Molander C, Hongpaisan J, Persson JKE (1994) Distribution of c-fos expressing dorsal horn neurons after electrical stimulation of low threshold sensory fibers in the chronically injured sciatic nerve. Brain Res 644:74-82.

Moore KA, Kohno T, Karchewski LA, Scholz J, Baba H, Woolf CJ (2002) Partial peripheral nerve injury promotes a selective loss of GABAergic inhibition in the superficial dorsal horn of the spinal cord. J Neurosci 22:6724-6731.

Okamoto M, Baba H, Goldstein PA, Higashi H, Shimoji K, Yoshimura M (2001) Functional reorganization of sensory pathways in the rat spinal dorsal horn following peripheral nerve injury. J Physiol (Lond) 532:241-250.

Polgár E, Fowler JH, McGill MM, Todd AJ (1999) The types of neuron which contain protein kinase $\mathrm{C}$ gamma in rat spinal cord. Brain Res 833:71-80.

Rexed B (1952) The cytoarchitectonic organization of the spinal cord in the cat. J Comp Neurol 96:415-496.

Riddell JS, Hadian M (1998) Topographical organization of neurones in group II reflex pathways of the rat spinal cord. J Comp Neurol 394:357-373.

Robertson B, Grant G (1985) A comparison between wheatgerm agglutinin and choleragenoid-horseradish peroxidase as anterogradely transported markers in central branches of primary sensory neurones in the rat with some observations in the cat. Neuroscience 14:895-905.

Scheibel ME, Scheibel AB (1968) Terminal axonal patterns in the cat spinal cord. II. The dorsal horn. Brain Res 9:32-58.

Shortland P, Woolf CJ (1993) Chronic peripheral nerve section results in a rearrangement of the central axonal arborizations of axotomized A beta primary afferent neurons in the rat spinal cord. J Comp Neurol 330:65-82.

Shortland P, Woolf CJ, Fitzgerald M (1989) Morphology and somatotopic organization of the central terminals of hindlimb hair follicle afferents in the rat lumbar spinal cord. J Comp Neurol 289:416-433.

Tong Y-G, Wang HF, Ju G, Grant G, Hökfelt T, Zhang X (1999) Increased uptake and transport of cholera toxin B-subunit in dorsal root ganglion neurons after peripheral axotomy: possible implications for sensory sprouting. J Comp Neurol 404:143-158.

Wilson P, Kitchener PD, Snow PJ (1996) Intraaxonal injection of Neurobiotin reveals the long-ranging projections of $\mathrm{A} \beta$-hair follicle afferent fibers to the cat dorsal horn. J Neurophysiol 76:242-254.

Woodbury CJ, Koerber HR (2003) Widespread projections from myelinated nociceptors throughout the substantia gelatinosa provide novel insights into neonatal hypersensitivity. J Neurosci 23:601-610.

Woodbury CJ, Ritter AM, Koerber HR (2000) On the problem of lamination in the superficial dorsal horn of mammals: a reappraisal of the substantia gelatinosa in postnatal life. J Comp Neurol 417:88-102.

Woolf CJ (1987) Central terminations of cutaneous mechanoreceptive afferents in the rat lumbar spinal cord. J Comp Neurol 261:105-119.

Woolf CJ (1997) Molecular signals responsible for the reorganization of the synaptic circuitry of the dorsal horn after peripheral nerve injury: the mechanisms of tactile allodynia. In: Molecular neurobiology of pain (Borsook D, ed) pp 171-200. Seattle: IASP.

Woolf CJ, Shortland P, Coggeshall RE (1992) Peripheral nerve injury triggers central sprouting of myelinated afferents. Nature 355:75-78. 